\title{
HELIOS: A helium line-ratio spectral-monitoring diagnostic used to generate high resolution profiles near the ion cyclotron resonant heating antenna on TEXTOR
}

E. A. Unterberg, O. Schmitz, D. H. Fehling, H. Stoschus, C. C. Klepper, J. M. Muñoz-Burgos, G. Van Wassenhove, and D. L. Hillis

Citation: Review of Scientific Instruments 83, 10D722 (2012);

View online: https://doi.org/10.1063/1.4739236

View Table of Contents: http://aip.scitation.org/toc/rsi/83/10

Published by the American Institute of Physics

\section{Articles you may be interested in}

Time-dependent analysis of visible helium line-ratios for electron temperature and density diagnostic using synthetic simulations on NSTX-U

Review of Scientific Instruments 87, 11E502 (2016); 10.1063/1.4955286

Hybrid time dependent/independent solution for the $\mathrm{He} I$ line ratio temperature and density diagnostic for a thermal helium beam with applications in the scrape-off layer-edge regions in tokamaks

Physics of Plasmas 19, 012501 (2012); 10.1063/1.3672230

Evaluation of thermal helium beam and line-ratio fast diagnostic on the National Spherical Torus ExperimentUpgrade

Physics of Plasmas 23, 053302 (2016); 10.1063/1.4948554

Spectroscopic imaging of limiter heat and particle fluxes and the resulting impurity sources during Wendelstein 7-X startup plasmas

Review of Scientific Instruments 87, 11D606 (2016); 10.1063/1.4959274

The Filterscope

Review of Scientific Instruments 74, 2068 (2003); 10.1063/1.1537038

Filterscopes: Spectral line monitors for long-pulse plasma devices

Review of Scientific Instruments 79, 10F330 (2008); 10.1063/1.2957777

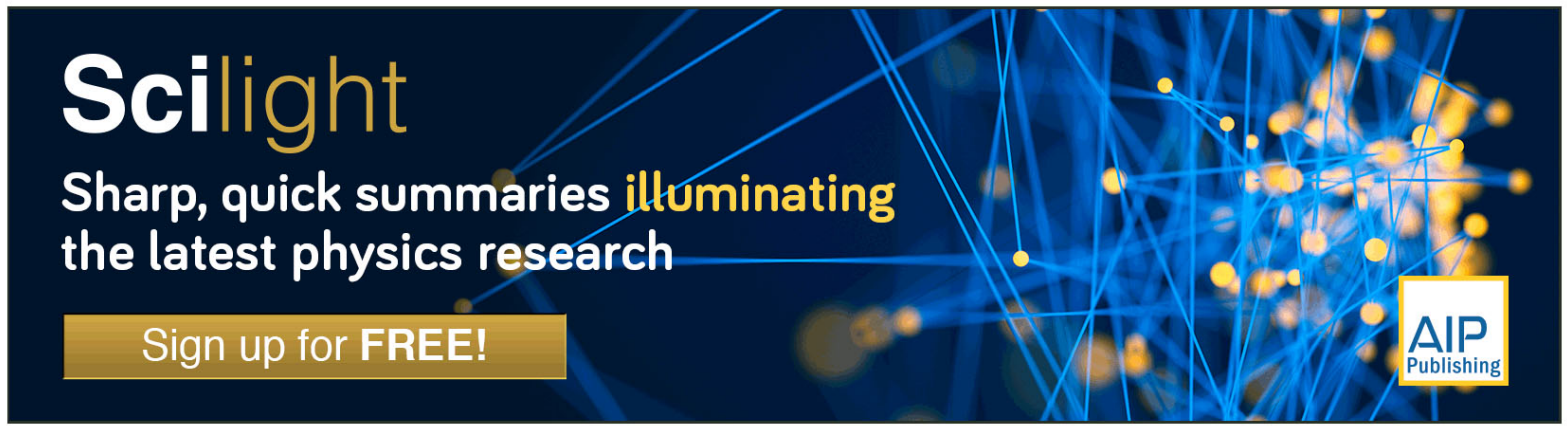




\title{
HELIOS: A helium line-ratio spectral-monitoring diagnostic used to generate high resolution profiles near the ion cyclotron resonant heating antenna on TEXTOR ${ }^{\text {a) }}$
}

\author{
E. A. Unterberg, ${ }^{1, b)}$ O. Schmitz, ${ }^{2}$ D. H. Fehling, ${ }^{1}$ H. Stoschus, ${ }^{2,3}$ C. C. Klepper,${ }^{1}$ \\ J. M. Muñoz-Burgos, ${ }^{3}$ G. Van Wassenhove, ${ }^{4}$ and D. L. Hillis ${ }^{1}$ \\ ${ }^{1}$ Oak Ridge National Laboratory, Oak Ridge, Tennessee 37831-6169, USA \\ ${ }^{2}$ Insitut für Energieforschung-Plasmaphysik, Forschungszentrum Jülich GmbH, Association EURATOM-FZJ, \\ D-52428 Jülich, Germany \\ ${ }^{3}$ Oak Ridge Institute for Science and Education, Oak Ridge, Tennessee 37831-0117, USA \\ ${ }^{4}$ LPP-ERM/KMS, Association EURATOM-Belgian State, B-1000 Brussels, Belgium
}

(Presented 8 May 2012; received 7 May 2012; accepted 10 July 2012; published online 1 August 2012)

\begin{abstract}
Radial profiles of electron temperature and density are measured at high spatial $(\sim 1 \mathrm{~mm})$ and temporal ( $\geq 10 \mu \mathrm{s}$ ) resolution using a thermal supersonic helium jet. A highly accurate detection system is applied to well-developed collisional-radiative model codes to produce the profiles. Agreement between this measurement and an edge Thomson scattering measurement is found to be within the error bars $(\lesssim 20 \%)$. The diagnostic is being used to give profiles near the ion cyclotron resonant heating antenna on TEXTOR to better understand RF coupling to the core. (C) 2012 American Institute of Physics. [http://dx.doi.org/10.1063/1.4739236]
\end{abstract}

\section{INTRODUCTION}

The helium line intensity ratio technique is a common method used to determine the radial profiles of electron temperature and density in the boundary region of magnetic fusion devices. Years of development with thermal beams of helium have led to their successful use to probe the plasma edge with minimal to no perturbation of the bulk plasma. ${ }^{1-3}$ These efforts require a detection system that is closely coupled to a collisional-radiative model (CRM) to accurately describe the physics processes of the thermal helium beam interacting with plasma. ${ }^{1,2,4,5}$

In this work, a remote and highly accurate detection system called "the filterscope" ${ }^{\prime, 7}$ is applied to a well-developed supersonic helium injector and CRM codes to produce high spatial $(\sim 1 \mathrm{~mm})$ and temporal $(\geq 10 \mu \mathrm{s})$ resolution radial profiles near an ion cyclotron resonant heating (ICRH) antenna ${ }^{8}$ on the TEXTOR tokamak. ${ }^{9}$ The addition of the filterscope system to the helium line-ratio technique adds unique capabilities to the method, e.g., it increases the signal-to-noise by individually detecting each emission line; it eliminates noise sources by placing the detector system well away from the device; and it allows simultaneous high sampling rates. This particular configuration is dubbed the helium line-ratio spectralmonitor (HELIOS) system as it uses narrow-bandpass filters to individually discriminate and sample each helium emission line. Details are presented describing the diagnostic hardware and technique as implemented on TEXTOR, and an overview

\footnotetext{
${ }^{a)}$ Contributed paper, published as part of the Proceedings of the 19th Topical Conference on High-Temperature Plasma Diagnostics, Monterey, California, May 2012.

b) Author to whom correspondence should be addressed. Electronic mail: unterberge@ fusion.gat.com.
}

of data obtained from the CRM models used with this setup is presented.

\section{APPARATUS}

A thermal supersonic helium jet is produced on TEXTOR and a 25 chord optical-train is aligned orthogonal to the jet to locally measure the intensities of three (3) helium emission lines at $667.8 \mathrm{~nm}, 706.5 \mathrm{~nm}$, and $728.1 \mathrm{~nm}$. The details of the choice of these emission lines are described in Sec. III. The full setup of the system is shown in Fig. 1. The figure shows, from left to right: a schematic of the TEXTOR machine hall; the diagnostic room rack that holds the detection hardware and control system; and the control room interface that is connected via ethernet to the rack. In the machine hall, a well-established Camparque-type supersonic injector system ${ }^{3}$ produces a neutral helium beam vertically, at $R=1.825 \mathrm{~m}$, and passes very near, $\sim 1 \mathrm{~cm}$, the upper edge of the ICRH antenna. This injector system is optimized for low divergence $\left( \pm 1^{\circ}\right)$ to minimize the line-of-sight integration across the beam axis. Field-line optics plus relay mirrors then focus the output of 25-chord fiber bundle at the observation volume (a $Z \simeq 5 \mathrm{~cm}$ by $R \simeq 10 \mathrm{~mm}$ volume). The fiber bundle then transfers the emission light outside the machine hall into a nearby diagnostic rack $(\sim 50 \mathrm{~m}$ from the collection optics). Each chord in the fiber bundle can terminate into a collimator coupled to one of twelve sets of detector assemblies inside the rack. A "patch panel" style interface allows easy configuration of the chords to the limited detector assemblies. Each assemble contains three pellicle beam splitters and a turning mirror. There is a narrow-bandpass $(\Delta \lambda \sim 1 \mathrm{~nm})$ 3 -cavity dichroic filter for each detection wavelength before the light enters compact photomultipler tubes (PMTs). Each PMT output goes through an anti-alising filter, gain amplifier, and buffer amplifier before going to the data acquisition. The 


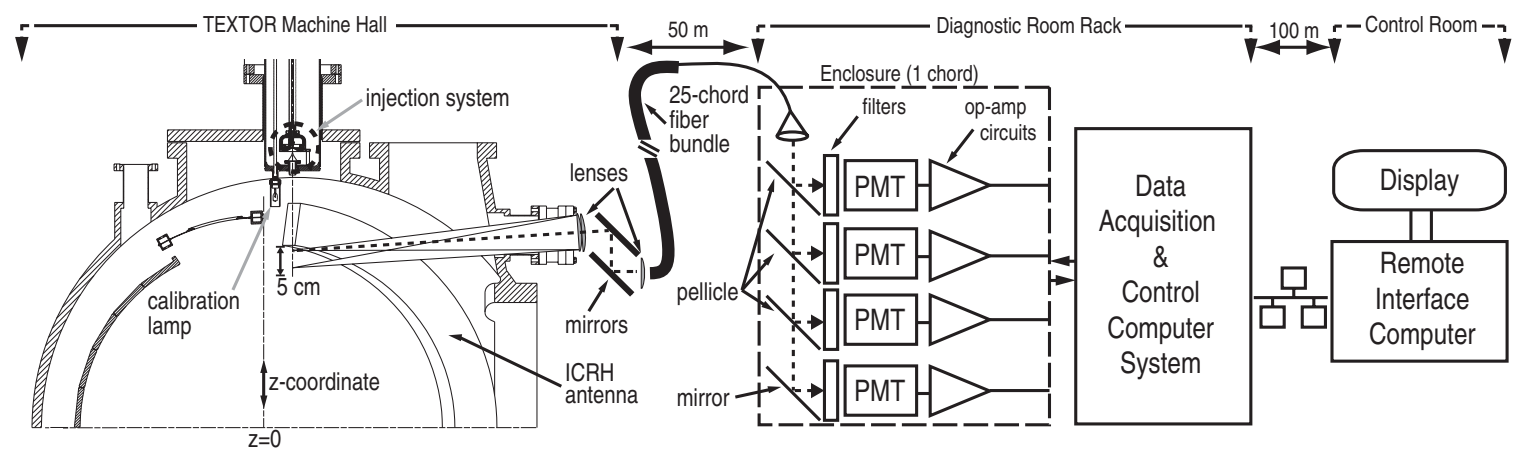

FIG. 1. A schematic diagram showing the general layout of the collection optics (left-hand side), detection hardware (middle), and the data acquisition and control (right-hand side).

details of the data acquisition and control can be found in Ref. 6. This equipment is remotely controlled via a local ethernet connection and data are archived and accessed on the local control computer via MDSplus. ${ }^{10}$

\section{RESULTS}

The details of the system of helium lines used in the CRMs for this application have been determined by many past studies. ${ }^{1,2,4,5,11}$ As CRM modeling and the measurements have improved, it has been determined that the emission lines from two singlet state transitions (at $667.8 \mathrm{~nm}$ and $728.1 \mathrm{~nm}$ ) and one triplet state transition (at $706.5 \mathrm{~nm}$ ) of neutral helium is sufficient to accurately determining the local electron temperature and density. The ratio of the $667.8 \mathrm{~nm}$ to $728.1 \mathrm{~nm}$ gives the electron density and the ratio of the $728.1 \mathrm{~nm}$ to $706.5 \mathrm{~nm}$ gives the electron temperature.
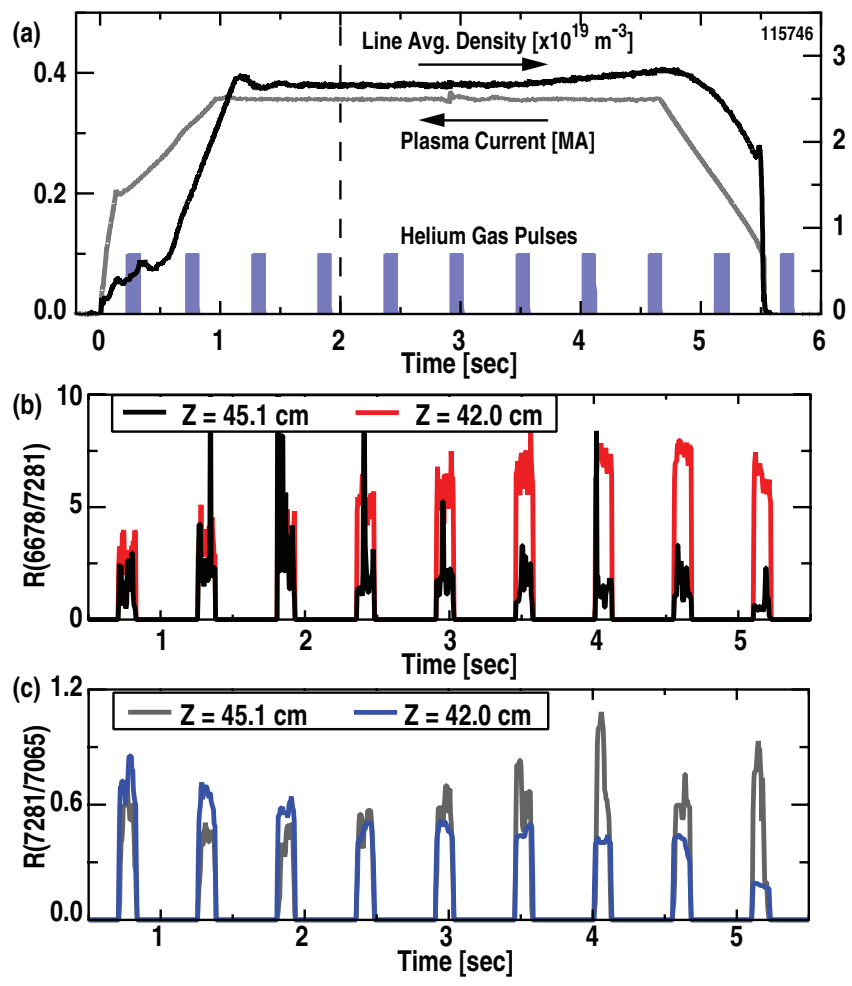

FIG. 2. The time history of a typical TEXTOR discharge with the HELIOS system. (a) shows the correlation of helium pulses within the discharge. (b) is the line-ratio of density for two viewchords versus time. (c) is the line-ratio of temperature for two viewchords versus time.
Figure 2 shows the time history of a discharge in TEXTOR with the HELIOS system. Figure 2(a) shows the plasma current and line-averaged, core density of the discharge. The discrete helium puffs are also shown in this subfigure for reference. The supersonic jet pulses at $2 \mathrm{~Hz}$ allowing the background signal to be subtracted before the ratio is taken. Each pulse has a $120 \mathrm{~ms}$ width. Two line ratios for the electron density $(R(6678 / 7281))$ from two viewchords are shown in Fig. 2(b) as a function of discharge time, and two helium line ratios for electron temperature $(R(7281 / 7065))$ from the same viewchords are shown in Fig. 2(c) as a function of discharge time. There are nine helium pulses (correlated with those seen in Fig. 2(a)) clearly seen in each of these subfigures. There are 12 chords of data spanning from well inside the last closed flux surface $(\sim 42 \mathrm{~cm})$ to near the ICRH antenna $(\sim 47 \mathrm{~cm})$.

The ratio data are analyzed with two well-developed and validated CRMs to determine the temperature and density profiles. The two CRMs used in this paper are termed the TEXTOR CRM and the hybrid CRM and are detailed in Refs. 2 and 5, respectively. The TEXTOR CRM is routinely used for helium line-ratio data on TEXTOR. It uses an equilibrium solution to the state populations to determine the temperature and density. The hybrid CRM is a newly developed and validated code that uses updated cross-section coefficients for the rate transitions and is capable of determining line-ratios with non-equilibrium state transitions.

The HELIOS measurement using both CRMs is compared with the TEXTOR Thomson scattering (TS) diagnostic during a recent ICRH campaign. Figures 3 and 4 show the profiles of electron temperature and density, respectively, from this comparison. The TS data were taken around $2 \mathrm{~s}$ as shown by the dashed line in Fig. 2(a), and the HELIOS data shown use the two helium pulses at $1.8 \mathrm{~s}$ and $2.3 \mathrm{~s}$ shown in Figs. 2(a) and 2(b). The data are sampled at $100 \mathrm{kHz}$. The profile data from the HELIOS system is averaged over each pulse for this comparison. The edge TS channels are shown in Figs. 3 and 4 with a spline fit was preformed to the whole TS profile in an effort to show the trend of the data when comparing to the HELIOS system. The error bars in the TEXTOR CRM are based on the standard deviation of the ratios within each pulse and are based on the variation in the line intensities for the hybrid CRM, see Ref. 5 for details. In both Figs. 3 and 4, the high spatial resolution of the HELIOS system $(\sim 1.4 \mathrm{~mm}$ chord-to-chord) can be seen. The large error bars on the 


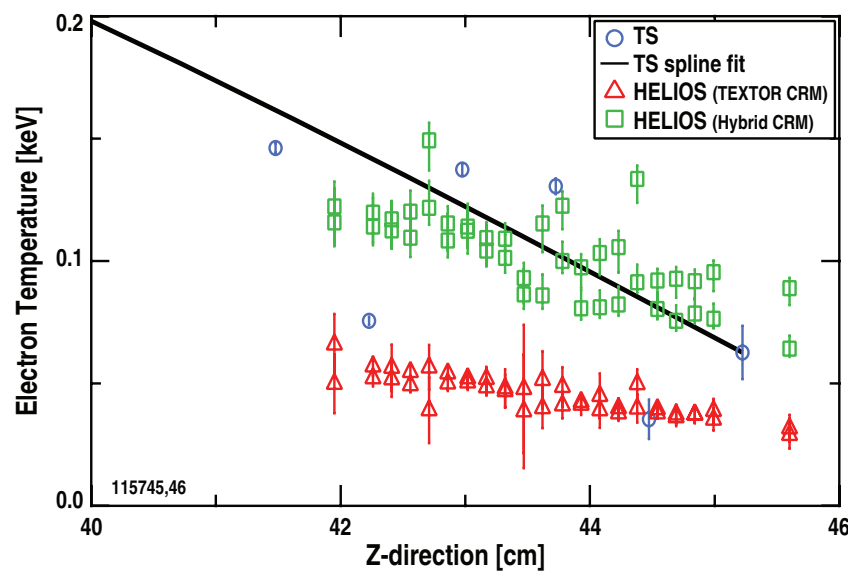

FIG. 3. Comparison of electron temperature profiles from TS and the HELIOS system using both the TEXTOR CRM and the hybrid CRM.

HELIOS system seen at $\sim 42.5 \mathrm{~cm}$ and $\sim 43.5 \mathrm{~cm}$ are due to noisy signals from the PMTs.

The hybrid CRM calculates the electron temperature $\sim 50 \mathrm{eV}$ higher than the TEXTOR CRM which compares better to the TS data and spline fit, which can be explained by use of time-dependence in the metastable triplet state in the CRM. ${ }^{5}$ Finally, there is a large discrepancy between the TS spline fit in density Fig. 4 versus the edge TS data. This coincides with the point where both CRM models predict the density profiles going from a flat profile to monotonically increase profile which indicates a limiter location. There is a possibility that $D_{\alpha}$ recycling light could be contaminating the

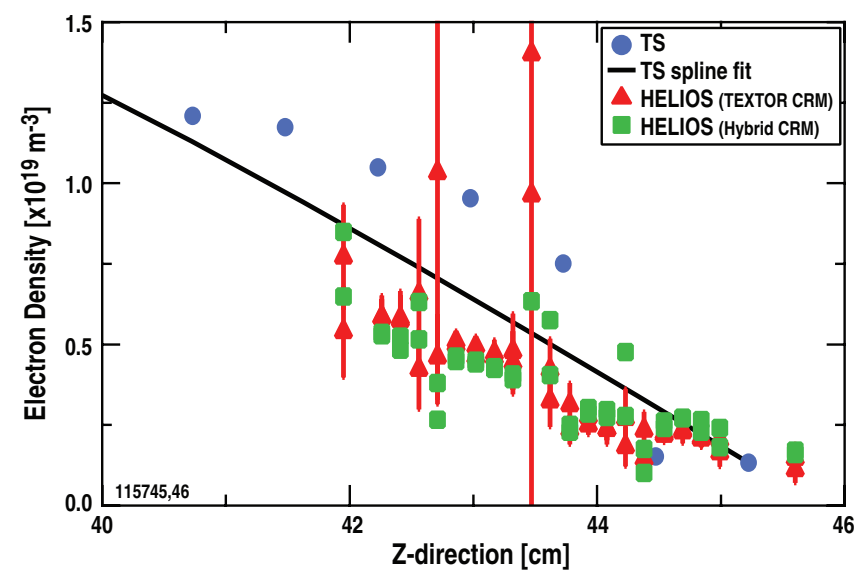

FIG. 4. Comparison of electron density profiles from TS and the HELIOS system using both the TEXTOR CRM and the hybrid CRM. raw TS data and causing an artificially high signal in the edge chords compared to the spline fit. ${ }^{12}$ Since the helium line ratio method is not susceptible to this recycling light, both HELIOS models are $\lesssim 20 \%$ of the TS spline fit when compared to the profile across the whole minor radius.

\section{DISCUSSION}

A new diagnostic system employing the helium line-ratio method has been installed on TEXTOR. This system uses a compact, portable, and easily expandable detection system coupled to a CRM for determining the radial profiles of electron temperature and density at high spatial and temporal resolution. Good agreement between this measurement and a TS measurement is found.

The system will be used during high power ICRH experiments to characterize the edge plasma and thereby aid the understanding of coupling RF heating power to the bulk plasma, as this coupling is highly dependent on the local electron parameters. Additionally, the fourth channel of each chord will be equipped with a $D_{\alpha}$ filter to determine the local neutral density when the main ion species is deuterium. This information is also important in determining the RF coupling to the core plasma.

\section{ACKNOWLEDGMENTS}

This work is sponsored by DOE Contract No. DE-AC0500OR22725. The authors would like to thank the ORNL Measurement Science and Systems Engineering Division and the TEXTOR operations team to ensure the success of the diagnostic.

${ }^{1}$ B. Schweer, M. Brix, and M. Lehnen, J. Nucl. Mater. 266, 673 (1999).

${ }^{2}$ O. Schmitz et al., Plasma Phys. Controlled Fusion 50, 115004 (2008).

${ }^{3}$ U. Kruezi, H. Stoschus, B. Schweer, G. Sergienko, and U. Samm, Rev. Sci. Instrum. 83, 065107 (2012).

${ }^{4}$ A. Pospieszczyk et al., Nucl. Instrum. Methods Phys. Res. B 72, 207 (1992).

${ }^{5}$ J. M. Muñoz-Burgos, O. Schmitz, S. D. Loch, and C. P. Ballance, Phys. Plasmas 19, 012501 (2012).

${ }^{6}$ R. J. Colchin, D. L. Hillis, R. Maingi, C. C. Klepper, and N. H. Brooks, Rev. Sci. Instrum. 74, 2068 (2003).

${ }^{7}$ N. H. Brooks et al., Rev. Sci. Instrum. 79, 10F330 (2008).

${ }^{8}$ G. van Oost et al., Fusion Technol. 12, 449 (1987).

${ }^{9}$ O. Neubauer et al., Fusion Sci. Technol. 47, 76 (2005).

${ }^{10}$ J. A. Stillerman, T. W. Fredian, K. A. Klare, and G. Manduchi, Rev. Sci. Instrum. 68, 939 (1997).

${ }^{11}$ A. Hidalgo, F. L. Tabarés, and D. Tafalla, Plasma Phys. Controlled Fusion 48, 527 (2006).

${ }^{12}$ H. J. van der Meiden et al., Rev. Sci. Instrum. 77, 10E512 (2006). 